\title{
Irregular Oscillations in a Realistic Abstract Quadratic Mass Action System *
}

\author{
K.-D. Willamowski and O. E. Rössler \\ Institute for Physical and Theoretical Chemistry, \\ University of Tübingen, West Germany
}

\section{Z. Naturforsch. 35a, 317-318 (1980); received December 24, 1979}

An open three-variable mass action kinetics is presented which exhibits chaotic behavior under numerical simulation. The elementary reactions of this system are at most of second order and satisfy the requirements of thermodynamics as long as the system is closed.

\section{Introduction}

In [1], Showalter et al. questioned the possibility of developing a realistic chemical mechanism which both satisfies the requirements of thermodynamics and exhibits "chaotic" behavior. It is the aim of this note to provide an example of such a mechanism which is based on at most second order elementary reactions.

\section{The System and its Dynamical Behavior}

Consider the following reaction mechanism

$$
\begin{aligned}
& A_{1}+X \underset{k_{-1}}{\stackrel{k_{1}}{\longrightarrow}} 2 X, \\
& X+Y \underset{\bar{k}_{2}}{\stackrel{k_{2}}{\longrightarrow}} 2 Y, \\
& A_{5}+Y \underset{k_{-3}}{\stackrel{k_{3}}{\rightleftarrows}} A_{2}, \\
& X+Z \underset{k_{-4}}{\stackrel{k_{4}}{\rightleftharpoons}} A_{3}, \\
& A_{4}+Z \underset{k_{-5}}{\stackrel{k_{5}}{\rightleftarrows}} 2 Z \text {. }
\end{aligned}
$$

The rate equations for system (1) under the usual conditions of isothermy, constant volume, and wellstirredness are:

$$
\begin{aligned}
\dot{x}= & k_{1} a_{1} x-k_{-1} x^{2}-k_{2} x y+k_{-2} y^{2}-k_{4} x z \\
& +k_{-4} a_{3}, \\
\dot{y}= & k_{2} x y-k_{-2} y^{2}-k_{3} a_{5} y+k_{-3} a_{2}, \\
\dot{z}= & k_{5} z a_{4}-k_{-5} z^{2}-k_{4} x z+k_{-4} a_{3}, \\
\dot{a}_{1}= & -k_{1} a_{1} x+k_{-1} x^{2}, \\
\dot{a}_{2}= & -k_{-3} a_{2}+k_{3} a_{5} y, \quad \dot{a}_{3}=-k_{-4} a_{3}+k_{4} x y, \\
\dot{a}_{4}= & -k_{5} a_{4} z+k_{-5} z^{2}, \quad \dot{a}_{5}=-k_{3} a_{5} y+k_{-3} a_{2} .
\end{aligned}
$$

* Presented at the Discussion Meeting "Kinetics of Physico-chemical Oscillations" of the Deutsche Bunsengesellschaft für Physikalische Chemie, Aachen 1979.

Reprint requests to Prof. O. E. Rössler, Institut für Physikalische und Theoretische Chemie, Universität Tübingen, Auf der Morgenstelle, D-7400 Tübingen.
The equilibrium composition (denoted by bars) of this closed system is easily obtained by solving the balance equations (left hand sides of Eq. (2) equal to zero)

$$
\begin{aligned}
k_{1} \bar{a}_{1} \bar{x} & =k_{-1} \bar{x}^{2}, \\
k_{2} \overline{x y} & =k_{-2} \bar{y}^{2}, \\
k_{3} \bar{a}_{5} \bar{y} & =k_{-3} \bar{a}_{2}, \\
k_{4} \overline{x z} & =k_{-4} \bar{a}_{3}, \\
k_{5} \bar{a}_{4} \bar{z} & =k_{-5} \bar{z}^{2} .
\end{aligned}
$$

It can be seen immediately that these five conditions are always fulfilled simultaneously and that the system meets the requirements of detailed balance regardless of the actual values of the rate constants. The same result can be obtained by applying Horn's [2] zero deficiency theorem to the system.

Suppose now that the concentrations of $A_{1}, \ldots, A_{5}$ are held constant exogeneously, thereby opening the system. This open system can be described by the following set of differential equations $(x=$ concentration of $X$, etc., $\cdot=\mathrm{d} / \mathrm{d} t)$ :

$$
\begin{aligned}
& \dot{x}=x\left(a_{1}-k_{-1} x-z-y\right)+k_{-2} y^{2}+a_{3}, \\
& \dot{y}=y\left(x-k_{-2} y-a_{5}\right)+a_{2}, \\
& \dot{z}=z\left(a_{4}-x-k_{-5} z\right)+a_{3},
\end{aligned}
$$

where all rate constants with the exception of $k_{-1}$, $k_{-2}$, and $k_{-5}$ are set equal to unity and $a_{1}$ through $a_{5}$ are the concentrations of the species held constant exogeneously.

\section{Nontrivial Dynamical Behavior of the Open System}

Choosing appropriate values for $a_{1}$ to $a_{5}$ and the rate constants $k_{-1}, k_{-2}$, and $k_{-5}$ in Eq. $\left(2^{\prime}\right)$, a rather complicated trajectorial behavior is obtained under numerical simulation, as seen in Figure 1. This behavior did not become periodic during simulation 


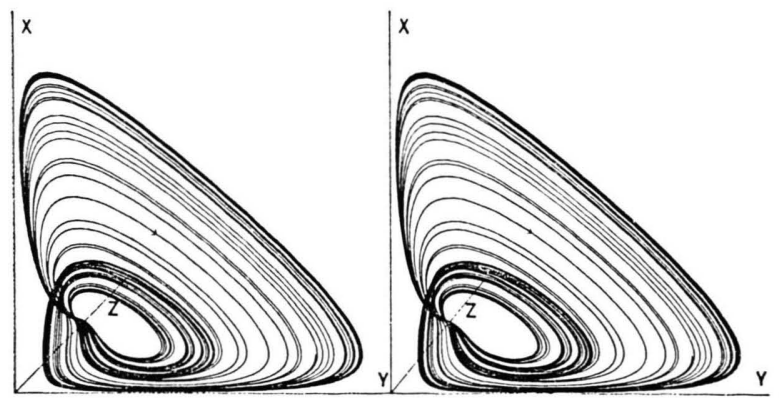

Fig. 1. Chaotic oscillations in Equation (2'). Stereoscopic display (two parallel projections). Parameters: $k_{-1}=0.25$, $k_{-2}=10^{-3}, k_{-5}=0.5, a_{1}=30, a_{2}=a_{3}=0.01, a_{4}=16.5$, $a_{5}=10$. Initial conditions: $x(0)=10, y(0)=80, z(0)=$ $0.1, t_{\mathrm{end}}=41.79$. Axes: $0 \ldots 100$ for $x$ and $y, 0 \ldots 50$ for $z$. Numerical simulation on a HP 9845 A desk computer.

times up to $t=1000$. The calculations were done using a fourth-order Runge Kutta Merson integration routine with automatic step-size control and low error bound $\left(10^{-7}\right.$ per finished step).

The proof that the system of Eq. (2') produces chaos in the mathematical sense [3] depends on the 2-dimensional Poincaré cross-section through the flow. If this cross-section is folded over at least once to a sufficient degree, there exist an infinite number of periodic solutions of different periodicities - almost all of them unstable - and an uncontable number of nonperiodic solutions. These two properties define chaotic flow. (See [4] for an historical review.) Such a multiply folded over cross-section was numerically found for the system of Eq. (2') [5].

Note that even for the system of Showalter et al. [1] it has not been excluded that there also exists an infinite number of unstable (saddle) limit cycles and an uncountable number of nonperiodic solutions besides the period- 6 limit cycle found. If these did exist, one could predict that the attracting period-6 limit cycle would disappear after a slight change of the parameters. It would be replaced by another imbedded attracting limit cycle of much larger period which cannot be detected numerically.

[1] K. Showalter, R. M. Noyes, and K. Bar-Eli, J. Chem. Phys. 69, 2514 (1979).

[2] F. Horn, Arch. Rat. Mech. Anal. 49, 172 (1972).

[3] T. Y. Li and J. A. Yorke, Amer. Math. Monthly 82, 985 (1975).

[4] O. E. Rössler, Chaos, in: Structural Stability in Physics (W. Güttinger, H. Eikemeier, eds.), SpringerVerlag, Berlin 1979, pp. $290-309$.

[5] K.-D. Willamowski and O. E. Rössler, in preparation.

[6] R. V. Plykin, Math. Sb. 94, 243 (1974).
We anticipate that the present system also contains a periodic attractor, even though we have not found it. It is unlikely that chaos-generating folded 2-dimensional maps without this property (like Plykin's map [6] can be realized in simple chemical systems [7].

\section{Discussion}

A recently described three-variable quadratic mass action system with (numerically) chaotic behavior [8] involved irreversible reactions and therefore was not chemically and thermodynamically realistic. It was of interest to check whether a related version, fulfilling the thermodynamic contraints imposed on realistic abstract reaction systems, still shows the same kind of behavior.

An investigation into the mathematical properties of the original version of system (1) (reactions 2, 3 , and 4 irreversible) [8] is presently in preparation [5]. Hereby, a rather wide range of parameter values has been found in which chaotic behavior can be expected theoretically and indeed occurs numerically. In light of these abstract results, the abovedescribed finding is not surprising, since the deviations from the more idealized equations are minor (small values for $k_{-2}, a_{2}$, and $a_{3}$ in the simulation of Figure 1).

It is known that realistic open two-variable quadratic mass action systems can only exhibit multistability and that three variables are necessary to obtain limit cycle oscillations [9]. This suggests a correlation between the number of independent variables and the dynamical behavior possible in second order mass action kinetics. Accordingly, one might have expected chaotic behavior to occur only in systems with at least four independent variables. The numerical results obtained here and in [8] surprisingly do not fulfill this expectation.

We thank Professor Richard M. Noyes for stimulating discussions. This work was supported by the Deutsche Forschungsgemeinschaft.

[7] O. E. Rössler, Chaos and Strange Attractors in Chemical Kinetics, in: Synergetics - Far from Equilibrium (A. Pacault, C. Vidal, eds.), SpringerVerlag, Berlin 1979, pp. 107-113.

[8] O. E. Rössler, Chaotic Oscillations in a 3-Variable Quadratic Mass Action System, in: Proc. Int'l. Symp. Math. Topics in Biology, Kyoto, Sept. 1978, pp. 131 135. Publ. Kyoto Research Institute for Math. Sci. Kyoto.

[9] P. Hanusse, C.R. Acad. Sci. Paris C 274, 1245 (1972). 